\title{
Sur un cas de myiase causé par Oestrus ovis dans une tannerie
}

\author{
par Gh. PASARE et I. VICEA \\ (Hôpital de la Ville, Turnu-Severin - Roumanie)
}

\begin{abstract}
Résumé
L'observation présentée rapporte un cas nouveau de myiase humaine due à Estrus ovis, constaté chez un employé d'une tannerie; les faits discutés ouvrent la voie à de nouveaux problèmes concernant la biologie de cet Insecte, ainsi que son cycle de vie parasitaire.
\end{abstract}

\section{Summary}

The observation here presents a new case of human myiase due to Estrus ovis, as seen on a worker in a tanninghouse; the facts discussed here open the way to new problems concerning the biology of this Insect, as well as its cycle-life as a parasite. 
Les cas de myiase due à CEstrus ovis sont peu connus en Roumanie. En effet, jusqu'à présent, la littérature de spécialité ne rapporte que trois communications à ce sujet. Ainsi, Iova (1965) relate deux cas de myiase oculaire chez des enfants, Nitzulescu et coll. publient un cas chez un adulte, trouvé en 1966 à Bucarest et Vicea, Pasăre et Petrescu décrivent, en 1967, un quatrième cas, toujours chez un enfant.

La plupart des cas humains décrits sont dus à l'attaque de la Mouche adulte ; ceux-ci évoluent surtout parmi des sujets qui ont un contact étroit avec les animauxhôtes habituels, c'est-à-dire les Moutons et les Chèvres.

Nos connaissances personnelles de l'évolution de la larve se limitent aux données classiques, selon lesquelles la mouche adulte dépose généralement les œufs ou les larves autour des narines des animaux; ces larves remontent passivement à l'intérieur des fosses nasales, étant entraînées par l'air inspiré, pour pénétrer ensuite de manière active dans les sinus du nez, où elles continuent leur évolution. A ce niveau, les larves se nourrissent soit de mucosités, soit du sang de la muqueuse pituitaire.

Chez l'homme, l'évolution des larves peut également avoir lieu au niveau du nez, mais le plus souvent on l'observe sur la conjonctive des globes oculaires, où elles peuvent provoquer des accidents très sérieux, qui exigent l'intervention médicale d'urgence, afin d'éviter une perte de la vue.

Dans un travail récent, J. Lapierre et M. Pette (1954) relatent un cas de myiase oculaire chez une femme engagée comme laveuse dans une boucherie, disposant d'une cour intérieure où l'on abattait différents animaux. La femme y a été contaminée pendant son travail. Les auteurs considèrent que la mouche adulte à incriminer provenait d'une larve qui avait glissé sur le sol après son évolution dans les sinus frontaux des animaux sacrifiés. Par conséquent, l'évolution de la larve jusqu'au stade de mouche adulte aurait eu lieu dans cette cour-même, ce qui constitue une condition subjective quasi-expérimentale pour la réalisation d'un cycle erroné de l'Insecte.

La constatation d'un second cas, dans l'intervalle d'un mois, toujours dans notre ville de Turnu-Séverin, mais cette fois-ci dans une tannerie centrale, donc à une distance d'environ $3 \mathrm{~km}$ de la campagne où se trouvaient les troupeaux de moutons, nous a décidés à faire la description de ce cas assez insolite.

Historique : Le $1^{\text {er }}$ septembre 1967, à 20 heures environ, l'ouvrier C. V..., âgé de 45 ans, s'est présenté à l'hôpital pour une consultation. A l'anamnèse, on note les faits suivants: l'ouvrier C. V... est employé dans la tannerie sus-mentionnée, où l'on tanne en quasi-exclusivité des peaux de moutons et de chèvres. Au moment où il prenait son repas de midi, dans la cour de l'entreprise, il a brusquement été heurté dans l'œil droit par un Insecte, immédiatement disparu après l'incident, donc avec impossibilité d'identifier sa nature. Quelques heures plus tard, il ressent des démangeaisons au niveau de cet œil, suivies de larmoiements, de gêne de la vue et de tuméfaction des paupières.

L'examen montre l'existence d'un chémosis intense ; à l'examen plus minutieux à l'aide d'une loupe on constate, dans le sac lacrymal et sur la conjonctive bulbaire, la 
présence de trois larves mobiles, ayant une longueur de 1,5-2 $\mathrm{mm}$, agités de mouvements vifs, flottant dans le liquide lacrymal. Les larves ont été immédiatement enlevées par lavage.

L'examen microseopique montre les caractères connus pour les larves d'Estrus ovis : anneau céphalique pourvu de deux crochets incurvés en forme de corne. Le corps est recouvert de poils latéraux situés sur les douze segments. Elle est de couleur blanche nacrée et offre des mouvements vifs sur lame. Par maintien dans l'éprouvette, la motilité est conservée pendant quelques heures. L'anneau terminal présente une plaque caractéristique en forme de lettre oméga, avec un nombre élevé de crochets (environ 20). Donc, il s'agissait - sans aucune équivoque — de larves d'Estrus ovis.

L'examen des fosses nasales du patient n'a pas mis en évidence la présence de larves à ce niveau.

\section{CONSTATATIONS PERSONNELLES :}

Ce cas, tout comme celui de Lapierre et Pette, nous a posé le problème de l'origine de cet Insecte dans la cour de la tannerie.

Par conséquent, nous nous sommes déplacés à cet endroit, où nous avons été surpris par le nombre considérable de mouches qui s'y trouvaient et qui rendaient presque insupportable l'activité des ouvriers qui y travaillaient. Par suite d'une pulvérisation immédiate avec des insecticides, nous avons tué un nombre impressionnant de mouches ; toutefois, nous n'avons pas réussi à déceler, parmi celles-ci, la mouche adulte qui nous intéressait.

Ensuite, nous avons procédé à l'inspection sommaire des dépôts pour les peaux de moutons et de chèvres venues de l'abattoir. Nous n'avons trouvé aucune nymphe d'Estrus ovis (qui sont plus facilement reconnaissables) sur le sol.

Nous soulignons le fait que les peaux des animaux - moutons et chèvres - sacrifiés et transportés à la tannerie n'avaient plus la partie qui recouvre la tête ; en effet, au niveau des narines et des yeux, celle-ci n'est que difficilement détachable des tissus.

\section{Discussion}

Dans un travail datant de 1938, Dinulescu, l'auteur qui - avec Coulombe - a décrit le premier cas de myiase chez l'homme provoquée par CEstrus ovis en Corse, s'occupe des Hypodermes de Roumanie. Tout en montrant les dégâts causés par ces Insectes dans l'industrie de la tannerie, l'auteur signale l'éventualité des myiases aussi chez l'homme aux niveaux des téguments, mais il ne cite pas de cas dus a Estrus ovis.

En ce qui concerne l'espèce Hypoderma qui est très voisine, aux points de vue bio'ogie et morphologie, d'Estrus ovis, le mécanisme de la ponte des œufs est relativement simple. La mouche dépose ses œufs sur les poils des animaux où les larves viennent à éclore et commencent leur migration pénétrante, soit par la peau, soit par voie buccale. 
Chez les Estrus ovis, les phénomènes biologiques du parasitisme diffèrent en quelque sorte : ici le phénomène de la pœcilogénie est rencontré en fonction du climat, la mouche déposant sur l'animal soit des œufs, soit des larves déjà écloses.

Dans le cas que nous décrivons, du fait que les peaux sont transportées, il est peu probable que la mouche adulte - malgré son instinct très développé de se maintenir autour de ces animaux - ait pu poursuivre le transport des peaux à travers la ville, c'est-à-dire de l'abattoir jusqu'à la tannerie. De même, la possibilité qu'elle ait été attirée par l'odeur des peaux de mouton dégagée par un troupeau du voisinage ne résiste également pas à un examen plus minutieux. Par conséquent, on peut de plano rejeter la probabilité de l'importation des mouches adultes et accepter la possibilité de leur introduction dans la tannerie en même temps que les peaux, sous la forme d'œufs ou de larves.

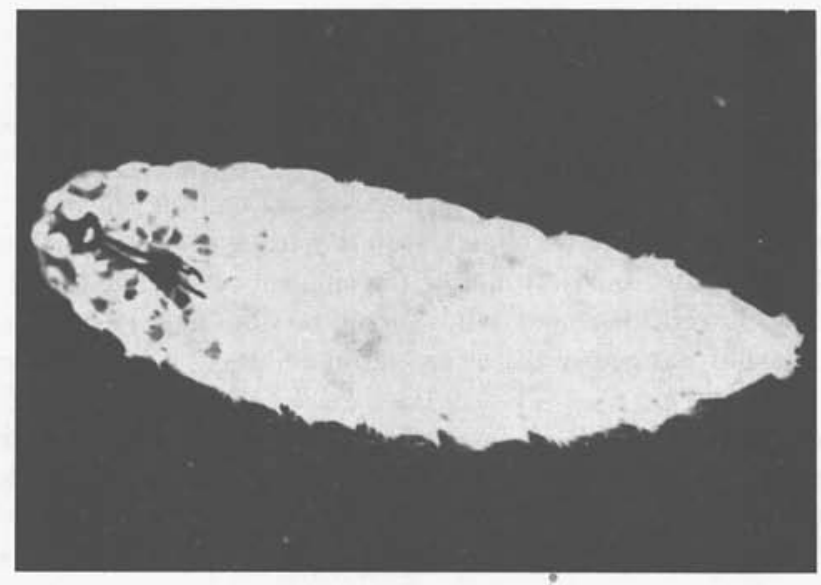

Larve vivante d'Cstrus ovis, avec crochets et plaque terminale

Le fait que la larve a continué à vivre et à se développer sur les peaux des animaux sacrifiés, s'y nourrissant donc, suggère l'idée que le cycle d'Estrus ovis, au cours de ses premiers stades, n'a pas lieu uniquement au niveau des fosses nasales et les sinus du nez du mouton ; il est probable qu'en conformité avec sa parenté ancestrale, la larve déposée sur la peau est capable de continuer son évolution, tout comme c'est le cas pour les Hypodermes. Cette constatation apparait sous la forme d'une impasse parasitaire (selon la conception de Harant) représentéa cette fois-ci par la peau de l'animal mort ; de cette impasse la mouche adulte dépose ses larves en passant par une seconde impasse: la conjonctive oculaire de l'homme.

Ceci explique l'attaque subie par notre patient, auquel elle s'est heurtée dans sa course folle pour trouver un hôte.

Il est indiscutab.e que l'essai de dépister (dans cette compétition des mouches qui déposent leurs œufs ou larves sur les peaux) les larves de mouches synantropes et les larves des CEstridés est un travail extrêmement laborieux; toutefois, il s'impose comme 
une recherche nécessaire dans l'avenir. Ce travail de déceler les larves d'Estridés sur la peau des moutons et des chèvres revient, obligatoirement dirons-nous, aux médecins vétérinaires.

L'observation que nous présentons suggère le fait que les Tabanidés ne sont pas les seuls à constituer un risque pour l'homme qui travaille dans les tanneries, mais également les Estridés, qui en sont des proches parents ; ceci, car aucune de ces deux espèces ne désavouent leur parenté, exprimée par un caractère phylogénétique commun, ce qui a conduit les hommes de science à les classer dans la même famille des Estridés.

\section{Références}

1. Brumpt (E.), 1948. - Précis de Parasitologie. Masson, Paris.

2. Dinulescu (Gh.), 1965. - Estridele. Ed. Academiei, Bucarest.

3. —, 1938. - Extras din revista Stiintelor Veterinare. Nr. 11-12, nov.-déc.

4. Iova (A.), 1965. - Pediatria, Bucarest, vol. 14, Nr. 1, p. 33-38.

5. Lapierre (J.), Pette (A.-N.), 1954. - Bulletin de la Soc. de Pathologie exotique, 47, $\mathrm{N}^{\circ}$ 4, p. 561-563.

6. - - - 1958. - Bulletin de la Soc. de Pathologie exotique, 51, $\mathrm{N}^{\circ} 2,232-238$.

7. Nitzulescu (V.), 1966. - Bulletin de la Soc. de Pathologie, XLI, $\mathrm{N}^{\circ} 4$, p. 379-386.

8. Vieulefont, Harant et Temple (de Montpellier), 1939. - Annales d'Oculistique.

9. Vicea (I.), Pasare (Gh.), Petrescu (G.). - Rev. Ophtalmologique (Bucarest), (sous presse). 